\title{
Early remodeling of rat cardiac muscle induced by swimming training
}

R.M.M. Verzola ${ }^{1}$, R.A. Mesquita ${ }^{1}$, S. Peviani ${ }^{1}$, O.H.P. Ramos ${ }^{1}$, A.S. Moriscot ${ }^{2}$, S.E.A. Perez ${ }^{1}$ and H.S. Selistre-de-Araújo ${ }^{1}$

\author{
${ }^{1}$ Departamento de Ciências Fisiológicas, Universidade Federal de São Carlos, \\ São Carlos, SP, Brasil \\ ${ }^{2}$ Departamento de Histologia e Embriologia, Instituto de Ciências Biomédicas, \\ Universidade de São Paulo, São Paulo, SP, Brasil
}

\section{Correspondence \\ H.S. Selistre-de-Araújo \\ Departamento de Ciências \\ Fisiológicas, UFSCar \\ Rodovia Washington Luís, km 235 \\ 13565-905 São Carlos, SP \\ Brasil \\ Fax: +55-16-3351-8327 \\ E-mail: hsaraujo@power.ufscar.br}

Publication supported by FAPESP.

Received July 18, 2005

Accepted January 2, 2006

\begin{abstract}
The aim of the present investigation was to study the effect of acute swimming training with an anaerobic component on matrix metallopeptidase (MMP) activity and myosin heavy chain gene expression in the rat myocardium. Animals (male Wistar rats, weighing approximately $180 \mathrm{~g}$ ) were trained for $6 \mathrm{~h} /$ day in 3 sessions of $2 \mathrm{~h}$ each for 1 to 5 consecutive days ( $N=5$ rats per group). Rats swam in basins 47 $\mathrm{cm}$ in diameter and $60 \mathrm{~cm}$ deep filled with water at 33 to $35^{\circ} \mathrm{C}$. After the training period a significant increase $(\mathrm{P}<0.05)$ was observed in the heart weight normalized to body weight by about 22 and $35 \%$ in the groups that trained for 96 and $120 \mathrm{~h}$, respectively. Blood lactate levels were significantly increased $(\mathrm{P}<0.05)$ in all groups after all training sessions, confirming an anaerobic component. However, lactate levels decreased $(\mathrm{P}<0.05)$ with days of training, suggesting that the animals became adapted to this protocol. Myosin heavy chain- $\beta$ gene expression, analyzed by real time PCR and normalized with GAPDH gene expression, showed a significant two-fold increase $(\mathrm{P}<0.01)$ after 5 days of training. Zymography analysis of myocardium extracts indicated a single $\sim 60-\mathrm{kDa}$ activity band that was significantly increased $(\mathrm{P}<0.05)$ after 72, 96, and $120 \mathrm{~h}$, indicating an increased expression of MMP-2 and suggesting precocious remodeling. Furthermore, the presence of MMP-2 was confirmed by Western blot analysis, but not the presence of MMP-1 and MMP-3. Taken together, our results indicate that in these training conditions, the rat heart undergoes early biochemical and functional changes required for the adaptation to the new physiological condition by tissue remodeling.
\end{abstract}

\section{Introduction}

The intrinsic ability of muscle to adapt during growth to endogenous stimuli and environmental signals such as exercise, hormonal changes, and electrical stimulation is the result of qualitative and quantitative changes in gene expression (1). Myosin is
Key words

- Myosin heavy chain

expression

- Training

- Real time PCR

- Rat myocardium

- Muscle hypertrophy the most abundant protein in muscle cells and the major component of the thick filament. It is considered to be a molecular motor that influences the rate of tension and fatigue development during muscle contraction (2). The myosin molecule is a complex 
formed by two heavy chains and two pairs of light chains that have an important role in modulating function during muscle contraction. The head region of the myosin heavy chain (MHC) contains the ATPase domain (3).

The existence of multiple isoforms of MHC enables the myosin motor to quickly adjust to widely different functional requirements (4). There are at least eight MHC isoforms in striated muscle whose mRNA and protein sequence are known: MHC-I/ß (slow isoform, expressed in skeletal muscle and heart, respectively), MHC-IIa, -Iib, and -IIx/IId (fast muscular isoforms, expressed in skeletal muscle), embryonic $\mathrm{MHC}$ and neonatal MHC (expressed during these life phases and during regeneration of adult skeletal muscle), and extraocular and mandibular MHC (expressed in these specific muscles). Skeletal MHC-I corresponds to the cardiac MHC-ß isoform (5).

A muscle fiber can alter its contractile properties by changing the absolute amounts of $\mathrm{MHC}$ isoforms. Exercise is a strong stimulus inducing this muscular adaptation to meet the imposed functional demands (6). Endurance training also induces transitions in the myosin isoform pattern which usually involve faster to slower isoform expression in skeletal muscle $(7,8)$. However, isoform transitions in the myocardium have been studied much less.

Collagen turnover in muscle is usually a dynamic process that involves both collagen synthesis and degradation (9). Collagen degradation depends on the activity of matrix metallopeptidases (MMPs), mostly collagenases (MMP-1) and later gelatinases (MMP-2 and MMP-9) (10). Regularly performed exercise such as long distance running and swimming results in cardiac hypertrophy in both experimental animals and man as a consequence of a maintained volume and pressure overload (11). In some studies, connective tissue hyperplasia occurred in the heart during the development of hypertro- phy, as a result of an increase in DNA and collagen concentration (12). However, little information is available regarding the effects of an intense and short training period in muscle remodeling.

The aim of this study was to analyze the short-term remodeling of the rat cardiac muscle during an acute training protocol by monitoring the expression of MHC- $\beta$ and MMP gene and protein expression. Our data show for the first time that up-regulation of the MHC gene may occur as early as after a 5-day period of intensive training, together with increased MMP-2 activity. These alterations could contribute to the precocious cardiac remodeling induced by this exercise protocol.

\section{Material and Methods}

\section{Animals and training protocol}

All animal procedures were done according to the Guide for Care and Use of Laboratory Animals (National Research Council, 1996, Guide for Care and Use of Laboratory Animals, National Academy Press, Washington, DC, USA). Adult male Wistar rats weighing about $180 \mathrm{~g}$ at the beginning of the procedure were used. Animals were submitted to swimming training for $6 \mathrm{~h} /$ day divided into three periods of $2 \mathrm{~h}$, with $30 \mathrm{~min}$ of rest between sessions (12). This protocol was chosen because it was shown to induce cardiac hypertrophy (12). Rats swam in basins $47 \mathrm{~cm}$ in diameter and $60 \mathrm{~cm}$ deep. The water temperature was controlled at 33 to $35^{\circ} \mathrm{C}$. All animals swan with weights (up to $20 \%$ body weight) fastened to the tail to avoid fluctuation, and an observer was present during the whole training sessions to confirm that the animals were really swimming.

The animals were divided into six groups of 5 rats each according to the duration of training ( 1 to 5 days). These groups will be referred to as the 24-, 48-, 72-, 96-, and 120-h 
groups. The control group ( $\mathrm{N}=5)$ was not trained (sedentary). Blood samples were collected from the caudal vein with heparincoated glass capillaries before and after each training session. Plasma lactate was measured with a lactate analyzer (1500 Sport, YSI, Yellow Springs, OH, USA). Sedentary and trained rats were sacrificed $24 \mathrm{~h}$ after the last training session. In a first group of animals, the hearts were excised and the left ventricle (including the septum) was dissected, weighed and frozen in liquid nitrogen. In a second set of experiments, the whole heart was excised, weighed and saved for standard histological evaluation, using $5-\mu \mathrm{m}$ thick sections stained with hematoxylin-eosin. Data are reported as means \pm SEM and were analyzed statistically by one-way analysis of variance (ANOVA). Statistical significance was evaluated by the Tukey post-test (comparing all groups) for $\mathrm{P}<0.05$.

\section{Preparation of total RNA}

Frozen cardiac tissue was homogenized and total RNA was isolated using cold Trizol Reagent (Invitrogen, Carlsbad, CA, USA) according to manufacturer instructions. Total RNA was quantified by spectrophotometry. RNA samples were treated with DNAse (Invitrogen) to avoid contamination with genomic DNA. All solutions were prepared with $0.01 \%$ DEPC-treated water, and glassware and plasticware were protected from contaminant RNase activity using standard procedures.

\section{cDNA synthesis and real time PCR}

cDNA synthesis was performed using the reverse transcriptase AMV (Promega Access Quick RT-PCR System, Madison, WI, USA). Real time PCR was performed using the SYBR Green Kit (Applied Biosystems, Foster City, CA, USA) and 10-ng samples. The GeneAmp 5700 Sequence Detection System software (Version 1.3; Ap- plied Biosystems) was used for quantitative PCR. All samples were analyzed in duplicate.

\section{Oligonucleotide primers}

Specific primers for the MHC- $\beta$ isoform (GenBank accession No. X15939) were used for real time PCR. The primer sequences were: MHC-I (ß) forward: 5'-AGAGAATGG CAAGACGGTGACT; reverse: 5'-CATGTC CTCGATCTTGTCGAACT (82-bp amplicon). A constitutive gene, glyceraldehyde3-phosphate dehydrogenase (GAPDH), was used to normalize the data using the same amount of cDNA. GAPDH primers were: forward: 5'-ATGCTGGTGCTGAGTATG TCG and reverse: 5' GTGGTGCAGGATG CATTGCTGA.

\section{Analysis of gene expression data}

To normalize the data for the control and trained groups, arbitrary units were calculated as: arbitrary unit $=2^{-\Delta \Delta \mathrm{TC}}$, and $\Delta \Delta \mathrm{TC}=$ sample $\Delta \mathrm{TC}$ - control $\Delta \mathrm{TC}$, where $\mathrm{TC}$ is the threshold cycle. Mean, standard deviation and one-way ANOVA were used for data analysis. Statistical significance was assessed by the Dunnett test, with the level of significance set at $\mathrm{P}<0.05$.

\section{Zymography}

Proteinase activity was detected on SDSgelatin gels (9). Briefly, frozen tissues (25 $\mathrm{mg}$ ) were washed with cold saline and incubated in $2 \mathrm{~mL}$ extraction buffer $(10 \mathrm{mM}$ cacodylic acid, pH 5.0, $150 \mathrm{mM} \mathrm{NaCl}, 1 \mu \mathrm{M}$ $\mathrm{ZnCl}_{2}, 20 \mathrm{mM} \mathrm{CaCl} 2,1.5 \mathrm{mM} \mathrm{NaN}_{3}$, and $0.01 \%$ Triton $\mathrm{X}-100$ ) at $4^{\circ} \mathrm{C}$ with continuous mixing for $24 \mathrm{~h}$. The $\mathrm{pH}$ was then raised to 7.5 with $1 \mathrm{M}$ Tris, $\mathrm{pH}$ 8.0. Total protein content was estimated using the BioRad dye binding assay (BioRad Laboratories, Hercules, CA, USA) according to the method of Bradford (13). For the enzymatic assay, SDS- 
gels $(15 \%)$ were prepared with $1 \mathrm{mg} / \mathrm{mL}$ gelatin and $30 \mu \mathrm{g}$ protein was loaded per lane without reduction. After electrophoresis, gels were washed twice for $15 \mathrm{~min}$ each with $2.5 \%$ Triton X-100 to eliminate SDS. Gels were then incubated overnight at $37^{\circ} \mathrm{C}$ in substrate buffer (50 mM Tris-HCl, $\mathrm{pH} 8.5,5 \mathrm{mM} \mathrm{CaCl}_{2}$, and $0.02 \% \mathrm{NaN}_{3}$ ). Gels were then stained for 30 min with $0.05 \%$ Coomassie blue R-250 in acetic acid:methanol:water (1:4:5) and destained in the same solution. All gels were prepared and run at the same time. The bands were quantified by densitometry using the Image Pro-plus software (Media Cybernetic, Silver Spring, MD, USA). Statistical significance was assessed by ANOVA followed by the Dunnett test. The enzymatic assays were also done in the presence of $15 \mathrm{mM}$ EDTA in the substrate buffer.

\section{Western blotting}

The same samples used for zymography were probed against anti-MMP-1, -2 , and -3 antibodies (Triple Point Biologics, Forest Grove, OR, USA) by Western blotting. Reduced samples were resolved by standard 15\% SDS-PAGE (14) and transferred to a nitrocellulose membrane. After blocking with $1 \%$ non-fat milk, the membrane was incubated with the first antibodies for $2 \mathrm{~h}$ at room temperature. Membranes were then washed and incubated with alkaline phosphatase- conjugated anti-IgG antibodies. BCIP and NBT (BioRad) were used as substrates for color development.

\section{Results}

\section{Body and heart weight and blood lactate levels}

A significant increase in body weight was evident after the fourth and fifth days of training compared with the group that was trained for 3 days but not compared to the control group (Table 1), probably due to a slight weight loss in the 72-h group. The heart weight was also significantly increased after 4 and 5 days of training (96-h and 120h groups) compared to control. Histological evaluation showed no alterations in the experimental groups compared to the control group (results not shown). Blood lactate levels were significantly increased after the training sessions in all trained groups compared to values obtained before the sessions for each day (Table 1, Figure 1). Animals seemed to adapt to training since blood lactate concentrations were reduced with the increase in the number of days of training before and immediately after all training sessions as well (Figure 1). Indeed, the blood lactate levels after training in the 120-h group were similar to the values of the 24-h group before training (Figure 1).

Table 1. Body and heart weights and blood lactate of rats before and after acute swimming training.

\begin{tabular}{llcccc}
\hline $\begin{array}{l}\text { Experimental } \\
\text { groups }\end{array}$ & $\begin{array}{l}\text { Initial/final } \\
\text { weight }(\mathrm{g})\end{array}$ & $\begin{array}{c}\Delta \mathrm{W} \\
\text { (final-initial } \\
\text { weight, } \mathrm{g})\end{array}$ & $\begin{array}{c}\text { Heart weight/ } \\
\text { body weight } \\
(\mathrm{mg} / \mathrm{g})\end{array}$ & $\begin{array}{c}\text { Blood lactate } \\
\text { before training } \\
(\mathrm{mmol} / \mathrm{L})\end{array}$ & $\begin{array}{c}\text { Blood lactate } \\
\text { after training } \\
(\mathrm{mmol} / \mathrm{L})\end{array}$ \\
\hline Control & $155.80 \pm 2.06 / 161.20 \pm 1.77$ & $5.4 \pm 1.21$ & $4.35 \pm 0.15$ & - & - \\
$72-\mathrm{h}$ & $161.80 \pm 2.54 / 161.40 \pm 4.06$ & $-0.4 \pm 1.54$ & $4.83 \pm 0.20$ & $3.18 \pm 0.43$ & $6.07 \pm 0.66^{*}$ \\
$96-\mathrm{h}$ & $167.40 \pm 3.56 / 179.80 \pm 6.20^{\mathrm{b}}$ & $12.4 \pm 4.7^{\mathrm{b}}$ & $5.32 \pm 0.10^{\mathrm{a}}$ & $2.09 \pm 0.23$ & $4.72 \pm 0.77^{*}$ \\
$120-\mathrm{h}$ & $175.40 \pm 4.74 / 191.00 \pm 7.29 \mathrm{~b}$ & $15.6 \pm 2.77^{\mathrm{b}}$ & $5.89 \pm 0.25^{\mathrm{a}, \mathrm{b}}$ & $1.70 \pm 0.18$ & $4.12 \pm 0.84^{*}$ \\
\hline
\end{tabular}

Data are reported as means \pm SEM for 5 rats per group.

aP $<0.05$ compared to control; bP $<0.05$ compared to the $72-\mathrm{h}$ group; ${ }^{*} \mathrm{P}<0.05$ compared to values before training within the same group (ANOVA followed by Tukey post-test). 
Myosin heavy chain isoform gene expression measured by mRNA levels

The changes in MHC- 8 mRNA levels occurred after 3 days of training and were statistically significant and more evident on the fifth day $(120 \mathrm{~h})$. At the end of the training protocol there was a significant increase in mRNA MHC- $\beta$ (Figure 2). Data for the 24-h, 48-h, and 72-h groups were dispersed and are not shown.

\section{Detection of matrix metallopeptidase activity}

MMP activity was detected in the myocardium extract as a single $\sim 60-\mathrm{kDa}$ lytic band (Figure 3A). This activity was completely abolished when the gels were incubated in substrate buffer with $15 \mathrm{mM}$ EDTA (results not shown). Increased MMP activity became statistically significant in the 72-h group, with a further increase in the 96-h and 120-h groups (Figure 3B). The appearance of lytic bands with molecular weights of approximately 62 and $58 \mathrm{kDa}$ suggested the activation of MMP-2, which was confirmed by Western blotting analysis with anti-MMP2 antibodies (Figure 3C). Anti-MMP-1 and -3 gave no signals in blots (results not shown); thus, MMP-2 was the only MMP activity detected in this condition.

\section{Discussion}

In the present study, we used a training protocol previously reported to induce cardiac hypertrophy (12) in order to identify the molecular mechanisms involved in this process. In fact, myocardium hypertrophy was suggested by the significant increase in heart weight after 96 and 120 h. Morphological evaluation showed no signs of edema or inflammatory infiltration, which could increase tissue weight, even in the absence of cellular growth. The increase of body weight at the end of training in the 96- and 120-h groups compared to the 72-h group corre- sponds to the physiological growth of rats. The lack of body weight gain in the first 3 days might be due to the stress during the adaptation period to the training protocol.

At the beginning of training ( $24 \mathrm{~h}$ ) blood lactate was high, probably due to the intensity of the protocol and to stress. These results indicate that this protocol has an anaerobic component. However, lactate levels before and after the sessions were progressively reduced with training, suggesting that animals became adapted.

The present results show that the slow myosin isoform MHC- $\beta$ gene was differently expressed in response to acute swimming training. Training induced significant up-regulation of the MHC- $\beta$ gene expression in the 120-h group compared to controls.

Both young and adult rats submitted to endurance training show a discrete increase
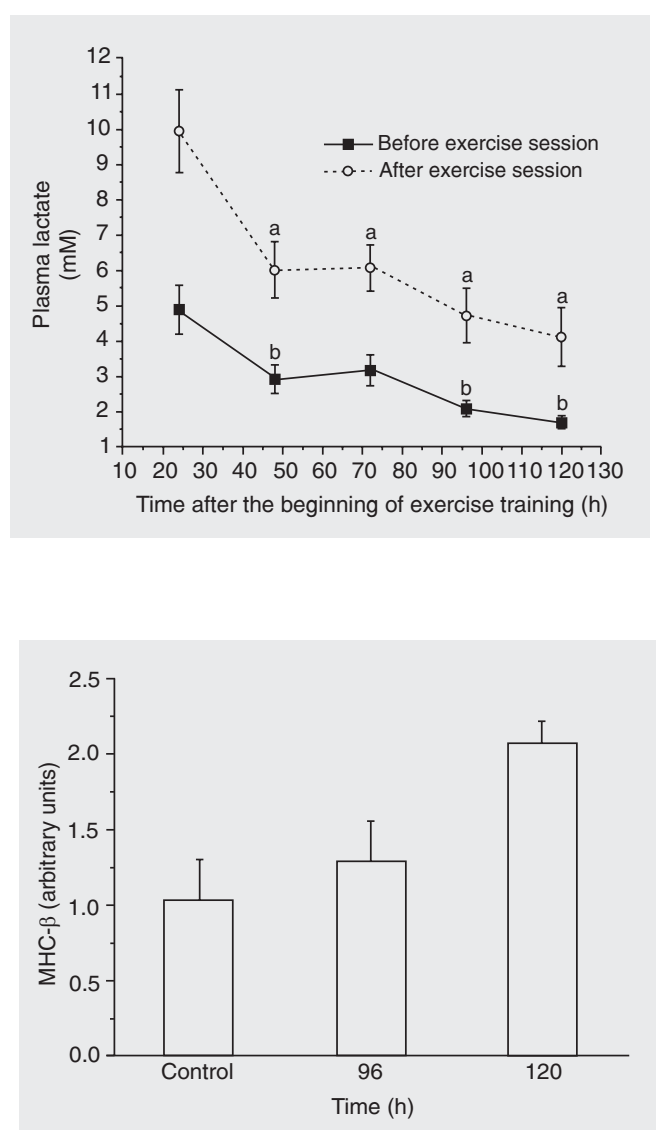

Figure 1. Adaptation of blood lactate concentration to training. Blood samples were taken before and after each training session. Each point is the mean value ( \pm SEM) for 5 animals. The symbols "a" and "b" represent data significantly different $(\mathrm{P}<$ 0.05 ) from the respective $24-h$ group after and before sessions, respectively (ANOVA followed by Tukey post-test).

Figure 2. Levels of MHC- $B$ gene expression in cardiac muscle before training (control), and after 96 and $120 \mathrm{~h}$ of training. MHC expression was normalized to GAPDH expression. $\mathrm{MHC}=$ myosin heavy chain. ${ }^{*} \mathrm{P}$ $<0.01$ compared to control (ANOVA followed by Dunnett post-test). 
in the expression of the MHC- $\beta$ gene, which is usually compensated by a similar decrease in the expression of MHC- $\alpha(15,16)$. Although these transformations are poorly understood, MacIntosh and colleagues (16) have shown that sympathectomized newborn rats have higher expression levels of MHC- $ß$ compared to controls. This observation suggests that in some way, the decrease in the activity of the sympathetic nervous system, which is a common adaptive re-

A
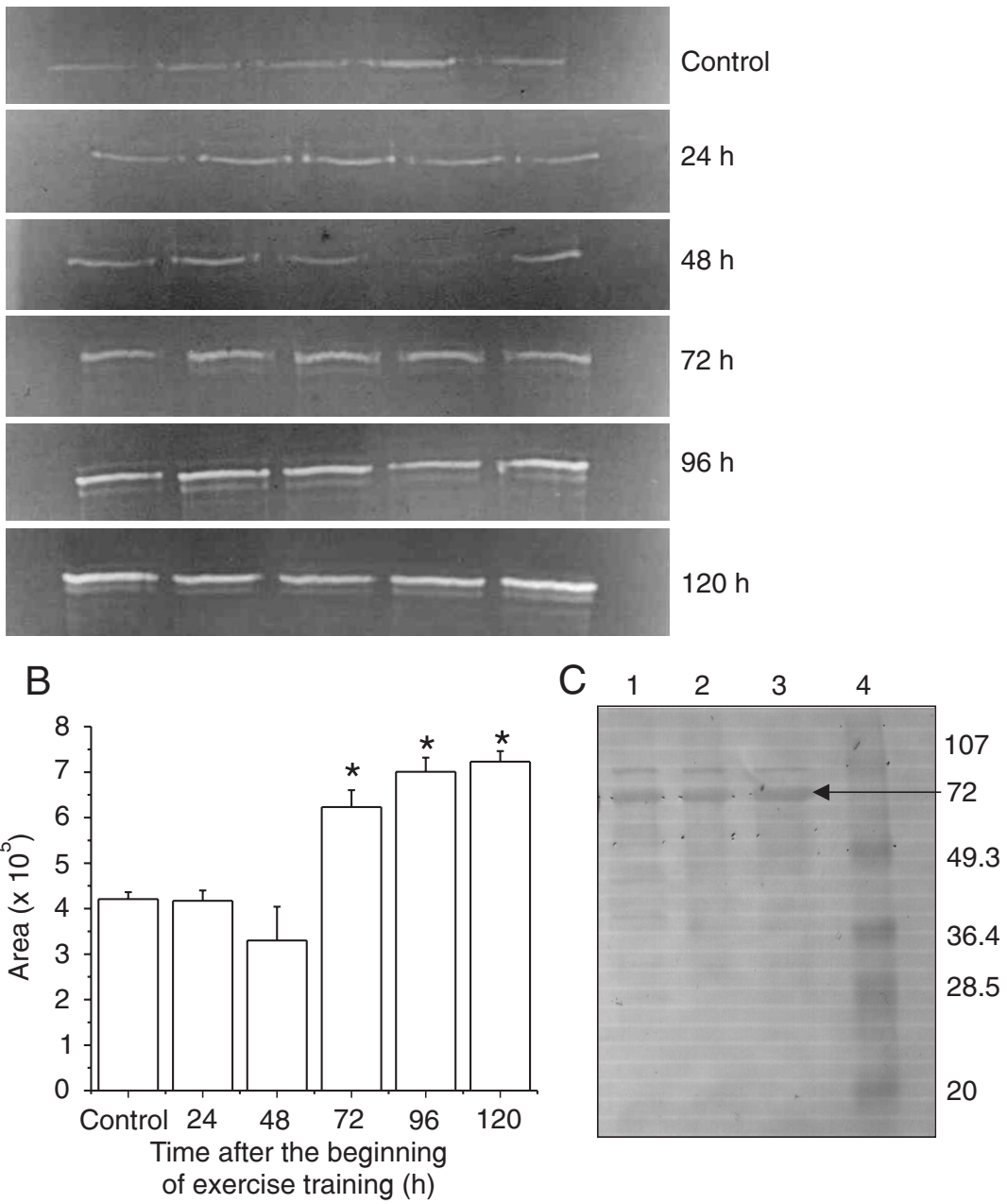

Figure 3. Detection of gelatinase in activity cardiac muscle extracts by zymography. After tissue extraction and SDS-PAGE with gelatin as a substrate, MMP-2 activity ( 60 kDa) was detected as an increase in gelatinase activity with training time. $A$, Each lane corresponds to an individual sample $(30 \mu \mathrm{g})$ from 1 animal for each group. $B$, The same bands were quantified by densitometry. ${ }^{*} \mathrm{P}<0.05$ compared to control (ANOVA followed by Dunnett post-test) $C$, Detection of MMP-2 by Western blotting. Proteins of cardiac tissue extracts were separated by $15 \%$ SDS-PAGE, transferred to a nitrocellulose membrane and probed with an anti-MMP-2 antibody. Lanes 1-3, Samples from 120-h group $(20 \mu \mathrm{g})$. Lane 4, Prestained SDS-PAGE Standard Low Range (Bio-Rad). MMP = matrix metallopeptidase. sponse to endurance training, may facilitate MHC- $\beta$ gene up-regulation.

We have shown here an increase in MHC$\beta$ expression using an acute experimental training protocol. Little is known about the myosin light chain gene expression in acute exercise. Using a different protocol in which rats swam 75 min twice daily, 5 days per week for 6 weeks, Buttrick et al. (17) did not observe modification in the expression of the myosin light chain I gene. However, the expression of MHC- $\beta$ and $-\alpha$ genes was not studied in that investigation.

The anaerobic threshold might be achieved and even exceeded in the swimming training protocol used here. If this is true, it could affect MHC- $\alpha$ and $-\beta$ expression. In fact, the results of blood lactate showed that the present protocol has an anaerobic component at least by the end of each training session, which could be an additional factor modifying gene expression. This is an interesting hypothesis that remains to be investigated.

The interventions that modify MHC expression result in economy of energy consumption during the process of power production to support the additional mechanical effort. Therefore, the heart can support the increased afterload associated with the hypertensive state as well as the volume overload due to endurance training $(18,19)$.

Myocardial remodeling was also suggested by a significant increase in gelatinase activity in the 72-, 96-, and 120-h groups. Gelatinase activity was completely abolished by EDTA, and the presence of MMP-2 was confirmed by Western blotting. The appearance of a lytic band of about $60 \mathrm{kDa}$ represents the activated form of this $72-\mathrm{kDa}$ gelatinase (9). No other proteins having MMP activity were observed in the heart muscle.

Heart remodeling is normally a dynamic process that involves both collagen synthesis and degradation (9). It has been demonstrated that MMPs exist in latent forms in the normal myocardium, and they can usually 
be activated in vitro by trypsin or plasmin (20). Increased MMP activity is necessary to permit tissue growth in order to support the increased metabolic needs during exercise.

The heart of rats can be biochemically and functionally remodeled depending on energy needs imposed not only on this tissue but on the whole body as well. These modifications are associated with the plasticity of MHC isoforms. At least in the heart, this early remodeling also involves the up-regulation and/or activation of MMP-2.

\section{Acknowledgments}

The authors would like to thank Dr. Vilmar Baldissera for critical discussion of the results as well as Jose Carlos Lopes, both from Departamento de Ciências Fisiológicas, Universidade Federal de São Carlos, São Carlos, SP, Brazil, and Antônio Garcia Junior, Departamento de Histologia e Embriologia, Instituto de Ciências Biomédicas, Universidade de São Paulo, São Paulo, SP, Brazil, for excellent technical assistance and skills.

\section{References}

1. Pette D (2001). Historical perspectives: plasticity of mammalian skeletal muscle. Journal of Applied Physiology, 90: 1119-1124.

2. O'Neill DS, Zheng D, Anderson WK et al. (1999). Effect of endurance exercise on myosin heavy chain gene regulation in human skeletal muscle. American Journal of Physiology, 45: 414-419.

3. Murphy AM (1996). Contractile protein phenotypic variation during development. Cardiovascular Research, 31: 25-33.

4. Reggiani C, Bottinelli R \& Stienen GJM (2000). Sarcomeric myosin isoforms: Fine tuning of a molecular motor. News in Physiological Sciences, 15: 26-33.

5. Eddinger TJ \& Meer DP (1997). Myosin isoform heterogeneity in single smooth muscle cells. Comparative Biochemistry and Physiology. Part B, Biochemistry and Molecular Biology, 117: 29-38.

6. Goldspink $G$ (1998). Selective gene expression during adaptation of muscle in response to different physiological demands. Comparative Biochemistry and Physiology. Part B, Biochemistry and Molecular Biology, 120: 27-39.

7. Baldwin KM \& Haddad F (2001). Effects of different activity and inactivity paradigms on myosin heavy chain gene expression in striated muscle. Journal of Applied Physiology, 90: 345-357.

8. Bigard XA, Janmot C, Merino D et al. (1996). Endurance training affects myosin heavy chain phenotype in regenerating fast-twitch muscle. Journal of Applied Physiology, 81: 2658-2665.

9. Cleutjens JPM, Kandala JC, Guarda E et al. (1995). Collagen remodeling after myocardial infarction in the rat heart. Journal of Molecular and Cellular Cardiology, 27: 1281-1292.

10. Docherty AJ \& Murphy G (1990). The tissue metalloproteinase family and the inhibitor TIMP: a study using cDNAs and recombinant proteins. Annals of the Rheumatic Diseases, 49: 469-479.
11. Scheuer J \& Tipton CM (1977). Cardiovascular adaptations to physical training. Annual Review of Physiology, 39: 221-251.

12. Hickson RC, Hammons GT \& Holloszy JO (1979). Development and regression of exercise-induced cardiac hypertrophy in rats. American Journal of Physiology, 236: 268-272.

13. Bradford MM (1976). A rapid and sensitive method for the quantitation of microgram quantities of protein utilizing the principle of protein-dye binding. Analytical Biochemistry, 72: 248-254.

14. Laemmli UK (1970). Cleavage of structural proteins during the assembly of the head of bacteriophage T4. Nature, 227: 680-685.

15. Swoap SJ, Haddad F, Bodell P et al. (1994). Effect of chronic energy deprivation on cardiac thyroid hormone receptor and myosin isoform expression. American Journal of Physiology, 266: 254-260.

16. Maclntosh AM, Mullin WM, Fitzsimons DP et al. (1986). Cardiac biochemical and functional adaptations to exercise in sympathectomized neonatal rats. Journal of Applied Physiology, 60: 991-996.

17. Buttrick PM, Kaplan M, Leinwand LA et al. (1994). Alterations in gene expression in the rat heart after chronic pathological and physiological loads. Journal of Molecular and Cellular Cardiology, 26: 61-67.

18. Alpert BS, Flood NL, Strong WB et al. (1982). Responses to ergometer exercise in a healthy biracial population of children. Journal of Pediatrics, 101: 538-545.

19. Swynghedauw B, Schwartz K, Lauer B et al. (1988). Striated muscle overload. European Heart Journal, 9: 1-6.

20. Tyagi SC, Ratajska A \& Weber KT (1993). Myocardial matrix metalloproteinase(s): localization and activation. Molecular and Cellular Biochemistry, 126: 49-59. 